\title{
Metástasis hepáticas sincrónicas de cáncer colorrectal. ¿Resección simultánea o diferida?
}

\section{Synchronous liver metastases from colorectal origin. Simultaneous or staged resection?}

\author{
Gustavo A. Nari*, Santiago López-Ben², Daniela Mariot', Maite Albio, Javier Góngora-Ortega³ y \\ Joan Figueras ${ }^{1}$ \\ ${ }^{1}$ Sección de Oncología Digestiva, Servicio de Cirugía General, Hospital Tránsito Cáceres de Allende, Córdoba, Argentina; ${ }^{2}$ Unidad de Cirugía Hepatobiliar \\ y Pancreática, Departamento de Cirugía, Hospital Josep Trueta, Gerona, España; ${ }^{3}$ Departamento de Estadística, ISEA, Aguascalientes, México
}

\begin{abstract}
Resumen
Introducción: El 20-40\% de las metástasis hepáticas de origen colorrectal son de tipo sincrónico. Actualmente existen tres estrategias quirúrgicas; dos de ellas proponen resecciones diferidas, y la otra, la resección simultánea. Objetivo: evaluar los resultados a corto y largo plazo de las resecciones simultáneas. Método: Evaluamos 212 metástasis hepáticas sincrónicas resecadas en dos centros y comparamos las intervenidas de forma simultánea con aquellas de manera diferida. Evaluamos las características demográficas, las resecciones hepáticas y las características de las metástasis. También evaluamos la morbimortalidad. Resultados: Fueron resecados de manera simultánea con el tumor primario 63 pacientes, y no hubo diferencias significativas en las características demográficas. Hubo más resecciones mayores $(p=0.005)$ en el grupo de las diferidas. La morbimortalidad fue comparable. La insuficiencia hepática $(p=0.037)$ fue mayor en el grupo de las diferidas. La morbilidad fue del $33.2 \%$ en las diferidas y del $10.1 \%$ en las simultáneas $(p=0.256)$. La mortalidad fue del $2.83 \%$ en las diferidas y del $0.94 \%$ en las simultáneas ( $p=0.508$ ). Conclusión: Los resultados a corto y largo plazo en ambos grupos son similares. Queda el interrogante de si la necesidad de una hepatectomía mayor favorecería la elección de un tratamiento diferido.
\end{abstract}

PALABRAS CLAVE: Metástasis colorrectales. Metástasis sincrónicas. Resección simultánea. Hepatectomías.

\begin{abstract}
Introduction: Between 20 and $40 \%$ of liver metastases from colorectal tumor are synchronous. Three types of surgical approaches are proposed; two of them propose a deferred resection and the other, simultaneous resection. The aim of this analysis is to assess the short- and long-term outcomes of simultaneous resections. Method: 212 synchronous liver metastases resected in two centers were evaluated. Comparison between those resected simultaneously with those that were in a deferred way was made. Demographics, liver resections and metastatic characteristics were evaluated. Morbidity and mortality of both alternatives are also evaluated. Results: 63 patients were resected simultaneously with the primary tumor, there were no significant differences in demographic characteristics. There was a greater number of major resections $(p=0.005)$ in the deferred group. Morbidity and mortality was comparable in both groups. Liver failure $(p=0.037)$ was higher in the deferred group. Morbidity was $33.2 \%$ in the deferred and $10.1 \%$ for the simultaneous $(p=0.256)$. Mortality rate was $2.83 \%$ in the deferred and $0.94 \%$ in the simultaneous group $(p=0.508)$. Conclusion: Short and long-term outcomes for both groups are similar. A question remains to be answered: the need of a major hepatectomy will favor the election of a deferred treatment?
\end{abstract}

KEY WORDS: Colorectal metastasis. Synchronous metastasis. Simultaneous resection. Hepatectomies.

\author{
Correspondencia: \\ *Gustavo Nari \\ Buchardo, 1200 \\ Fecha de recepción: 27-03-2018 \\ 5000 Córdoba, Argentina \\ Fecha de aceptación: 20-07-2018 \\ E-mail: gusnari@ hotmail.com \\ DOI: 10.24875/CIRU.18000312
}

Cir Cir. 2018;86:528-533

Contents available at PubMed

www.cirugiaycirujanos.com 


\section{Introducción}

Las resecciones hepáticas con intención curativa son en la actualidad la mejor opción para los pacientes con metástasis de origen colorrectal. Alrededor del $50 \%$ de los pacientes con cáncer colorrectal desarrollarán metástasis hepáticas en el transcurso de su evolución'. De todas ellas, un 20 a 50\% lo harán de manera sincrónica ${ }^{2-5}$. Los avances en cuidados intensivos, anestesiología, conocimiento de la anatomía y de la fisiología hepática, tecnología en imágenes y sección parenquimatosa, así como el desarrollo de nuevos agentes quimioterápicos, han ampliado las indicaciones quirúrgicas de estos pacientes $^{6-11}$.

Hasta hace unos años, la presencia de metástasis sincrónicas de hígado de origen colorrectal se resolvía actuando en primer lugar sobre el tumor colorrectal y posteriormente quimioterapia, y en los pacientes con buena respuesta se indicaba la extirpación de las metástasis hepáticas ${ }^{12,13}$. La asociación de los adelantos antes mencionados y el conocimiento de que los regímenes contemporáneos de quimioterapia desencadenaron algunos efectos secundarios que aumentan la morbilidad y la mortalidad después de la resección (esteatosis, esteatohepatitis, dilatación sinusoidal y congestión) $)^{14-16}$ han favorecido la propuesta de las resecciones simultáneas del tumor primario y las metástasis hepáticas.

El objetivo del presente trabajo es evaluar los resultados a corto y largo plazo de las resecciones simultáneas en pacientes con metástasis sincrónicas de hígado de origen colorrectal, con el convencimiento de que no existen diferencias cuando se comparan con aquellas que se realizan de manera diferida.

\section{Método}

Se analizan los datos de pacientes sometidos a resección hepática por metástasis sincrónicas de hígado de origen colorrectal en dos centros (Hospital Tránsito Cáceres de Allende y Hospital Josep Trueta) entre los años 1997 y 2013 (16 años). Se comparan los resultados de los pacientes que fueron sometidos a resecciones simultáneas con aquellos en los que la resección se hizo de manera diferida. Los datos fueron recogidos prospectivamente y analizados de manera retrospectiva. Los pacientes con hepatectomías en dos tiempos fueron excluidos del análisis, al igual que aquellos con enfermedad extrahepática que no podía ser resecada en su totalidad. Se recogieron las características demográficas estándar y clínico-patológicas, así como los datos referentes a las hepatectomías. Todos los pacientes que recibieron quimioterapia perioperatoria en los últimos años tuvieron un esquema basado en oxiplatino e irinotecán.

\section{Resecciones hepáticas}

Se consideraron resecables todos aquellos pacientes en quienes la cirugía podía extirpar toda la masa tumoral hepática, independientemente del tamaño y del número de metástasis en el hígado. Se definió como resección mayor la que involucró tres o más segmentos consecutivos de la clasificación de Couinaud. La nomenclatura utilizada es la propuesta en Brisbane $^{17}$. La transección del parénquima hepático fue realizada por diferentes procedimientos, que comprendieron el uso de kellyclasia, bisturís monopolares y bipolares, bisturís armónicos y Compact Ultrasonic Surgical Aspiration (CUSA). El tiempo de resección hepática se midió en minutos desde el inicio de la incisión hasta el cierre definitivo de la piel del paciente.

\section{Resultados a corto plazo}

Se consideraron como mortalidad posoperatoria aquellos pacientes que fallecieron dentro de los primeros 30 días, durante la hospitalización o en un reingreso por complicaciones inherentes a la cirugía. La morbilidad se definió como cualquier evento adverso inherente al procedimiento quirúrgico, y se clasificó de acuerdo con la propuesta de Dindo-Cla$v_{i e n}{ }^{18}$. La insuficiencia hepática se definió según el criterio «50-50»19. Las pérdidas se midieron en mililitros. La estancia hospitalaria se estableció en días.

\section{Seguimiento posoperatorio}

Los pacientes fueron seguidos de manera regular con controles de laboratorio, marcadores tumorales y diagnóstico por imágenes. El seguimiento y el control de sobrevida se hizo hasta la muerte del paciente 0 hasta la fecha del último control en consultorio. La sobrevida se determinó a 5 años y se midió la supervivencia libre de enfermedad. Se evaluaron los siguientes factores pronóstico: localización rectal del tumor primario, presencia de ganglios positivos, presencia de enfermedad extrahepática, localización 
bilobar de las metástasis y presencia de más de tres lesiones.

\section{Análisis estadístico}

Las variables categóricas se compararon con el test de ji al cuadrado, y las variables continuas con el sistema ANOVA. En el análisis de sobrevida se utilizó la prueba de Kaplan-Meier y las diferencias en las curvas de supervivencia se compararon usando el test de Log-rank. El análisis estadístico se hizo con el sistema SPSS versión 20, y se consideró significativa una $p<0.05$.

\section{Resultados}

Entre 1997 y 2013 se realizaron 599 resecciones hepáticas con intención curativa por metástasis de origen colorrectal; de ellas, 212 fueron sincrónicas (35.4\%) y constituyen el universo de estudio. Fueron intervenidos de manera diferida o clásica (resección colorrectal, quimioterapia y hepatectomía) 149 pacientes $(70.3 \%)$, y de manera simultánea (hepatectomía seguida de resección colorrectal en el mismo acto operatorio) 63 pacientes (29.7\%).

Hubo predominio del sexo masculino. La localización colónica fue más frecuente que la rectal, al igual que el tipo Dukes $\mathrm{C}$ y el estadio pT3. El número de ganglios positivos en ambos grupos de estudio no presentó diferencias significativas, al igual que los marcadores tumorales antes de la hepatectomía. En la Tabla 1 se detallan las variables demográficas y las características del tumor primario.

En la Tabla 2 se presentan las variables correspondientes a las resecciones hepáticas y las características de las metástasis. En ella puede observarse que hubo más resecciones mayores en el grupo de pacientes tratados de manera clásica, con una diferencia estadísticamente significativa $(p=0.005)$. La presencia de enfermedad bilobar o de enfermedad extrahepática, el número de metástasis, el margen de resección y las pérdidas hemáticas no mostraron diferencias estadísticas entre ambos grupos.

La morbilidad de las resecciones diferidas fue del $33.2 \%$, mientras que la de las resecciones simultáneas fue del $10.1 \%(p=0.256)$. La mortalidad en el grupo de pacientes tratados de manera clásica fue del $2.83 \%$, en tanto que en el de cirugía simultánea fue del $0.94 \%(p=0.508)$.

Dentro de las complicaciones que pueden verse en la Tabla 3, la insuficiencia hepática presentó diferencias
Tabla 1. Variables demográficas

\begin{tabular}{|c|c|c|c|}
\hline Variable & $\begin{array}{l}\text { Resección } \\
\text { simultánea }\end{array}$ & $\begin{array}{l}\text { Resección } \\
\text { diferida }\end{array}$ & $\mathrm{p}$ \\
\hline Edad & 63.5 años & 63.0 años & 0.619 \\
\hline Sexo masculino & $69.76 \%$ & $70.24 \%$ & 0.544 \\
\hline Tumor primario recto & $8.86 \%$ & $23.14 \%$ & 0.086 \\
\hline Dukes A & $0.61 \%$ & $1.84 \%$ & 0.823 \\
\hline Dukes B & $3.68 \%$ & $21.47 \%$ & 0.826 \\
\hline Dukes C & $12.88 \%$ & $59.50 \%$ & 0.865 \\
\hline pT1 & $0.56 \%$ & $1.12 \%$ & 0.883 \\
\hline pT2 & $1.12 \%$ & $3.91 \%$ & 0.874 \\
\hline pT3 & $11.73 \%$ & $57.54 \%$ & 0.515 \\
\hline Ca19-9 prehepatectomía & $16.92 \mathrm{U} / \mathrm{ml}$ & $83.08 \mathrm{U} / \mathrm{ml}$ & 0.272 \\
\hline CEA prehepatectomía & $16.23 \mathrm{ng} / \mathrm{ml}$ & $83.76 \mathrm{ng} / \mathrm{ml}$ & 0.914 \\
\hline $\mathrm{N+}$ & $10.92 \%$ & $55.74 \%$ & 0.672 \\
\hline pNO & $6.18 \%$ & $26.40 \%$ & 0.769 \\
\hline $\mathrm{pN1}$ & $6.18 \%$ & $29.21 \%$ & \\
\hline pN2 & $4.49 \%$ & $27.52 \%$ & \\
\hline
\end{tabular}

Tabla 2. Características de las metástasis y de las resecciones hepáticas

\begin{tabular}{lccc}
\hline Variable & $\begin{array}{c}\text { Resección } \\
\text { simultánea }\end{array}$ & $\begin{array}{c}\text { Resección } \\
\text { diferida }\end{array}$ & $\mathbf{p}$ \\
\hline Hepatectomía mayor & $2.83 \%$ & $28.77 \%$ & 0.005 \\
Tamaño & $4.23 \mathrm{~cm}$ & $3.39 \mathrm{~cm}$ & 0.199 \\
Bilobar & $5.5 \%$ & $36 \%$ & 0.275 \\
Número de metástasis & 2.81 & 3.98 & 0.595 \\
Enfermedad extrahepática & $4.48 \%$ & $14.43 \%$ & 0.116 \\
Margen & $0.96 \mathrm{~cm}$ & $0.71 \mathrm{~cm}$ & 0.218 \\
Pérdidas & $552 \mathrm{ml}$ & $767 \mathrm{ml}$ & 0.147 \\
Duración cirugía & $239.8 \mathrm{~min}$ & $242.8 \mathrm{~min}$ & 0.874 \\
Oclusión portal & $1.41 \%$ & $10.85 \%$ & 0.179 \\
Estancia hospitalaria & $11.6 \mathrm{días}$ & $9.4 \mathrm{días}$ & 0.187 \\
Morbilidad & $10.09 \%$ & $33.17 \%$ & 0.256 \\
Mortalidad & $0.94 \%$ & $2.83 \%$ & 0.508 \\
\hline
\end{tabular}

significativas entre ambos grupos ( $p=0.037$ ), con un franco predominio en las resecciones diferidas (9.9\%). El resto de las complicaciones no mostraron diferencias entre ambos grupos. En el análisis de la gravedad de la complicación, la mayoría pertenecían a los grupos 2 y 3 de la clasificación de Dindo-Clavien (Tabla 4). En referencia a la evaluación de los factores pronóstico, la 
Tabla 3. Complicaciones de las hepatectomías

\begin{tabular}{lccc}
\hline Complicación & $\begin{array}{c}\text { Resección } \\
\text { simultánea }\end{array}$ & $\begin{array}{c}\text { Resección } \\
\text { diferida }\end{array}$ & $\mathbf{p}$ \\
\hline Fístula biliar & $1.89 \%$ & $3.77 \%$ & 0.208 \\
Insuficiencia hepática & $0.47 \%$ & $9.90 \%$ & 0.037 \\
Absceso intraabdominal & $3.30 \%$ & $11.32 \%$ & 0.446 \\
Ascitis & $1.44 \%$ & $7.69 \%$ & 0.440 \\
Hemoperitoneo & $0.94 \%$ & $6.60 \%$ & 0.332 \\
Infección & $1.89 \%$ & $5.19 \%$ & 0.361 \\
Neumopatía & $1.42 \%$ & $2.83 \%$ & 0.267 \\
\hline
\end{tabular}

Tabla 4. Gravedad de las complicaciones

\begin{tabular}{lccc}
\hline Grado de complicación & $\begin{array}{c}\text { Resección } \\
\text { simultánea }\end{array}$ & $\begin{array}{c}\text { Resección } \\
\text { diferida }\end{array}$ & $\mathbf{p}$ \\
\hline Grado 1 & $3.37 \%$ & $12.35 \%$ & 0.202 \\
Grado 2 & $4.49 \%$ & $21.35 \%$ & \\
Grado 3 & $14.60 \%$ & $26.96 \%$ & \\
Grado 4 & $1.12 \%$ & $7.86 \%$ & \\
Grado 5 & $0 \%$ & $7.86 \%$ & \\
\hline
\end{tabular}

localización rectal del tumor primario $(p=0.842)$, la presencia de ganglios positivos en la resección intestinal $(p=0.147)$, la localización bilobar $(p=0.203)$ y un número mayor de tres lesiones $(p=0.562)$ no resultaron ser factores de mal pronóstico, mientras que la presencia de enfermedad extrahepática $(p=0.048)$ sí resultó ser un factor de mal pronóstico.

Finalmente, en el análisis de la supervivencia a 5 años y de la supervivencia libre de enfermedad (Figs. 1 y 2 ) no hubo diferencias ( $p=0.254$ y $p=0.348$, respectivamente).

\section{Discusión}

Alrededor del $50 \%$ de los pacientes con cáncer colorrectal desarrollarán metástasis hepáticas en algún momento de su evolución. Entre un 20 y un $40 \%$ de ellas serán sincrónicas. El abordaje clásico de estos pacientes ha sido la resección del tumor primario y posteriormente realización de quimioterapia y resección hepática de las metástasis con intención curativa. La aparición de nuevos agentes quimioterápicos y sus consecuencias sobre el hígado, asociado a una franca mejoría en los resultados de la cirugía hepática, favorecieron la propuesta de la cirugía simultánea del tumor y las metástasis hepáticas. Por otra parte,

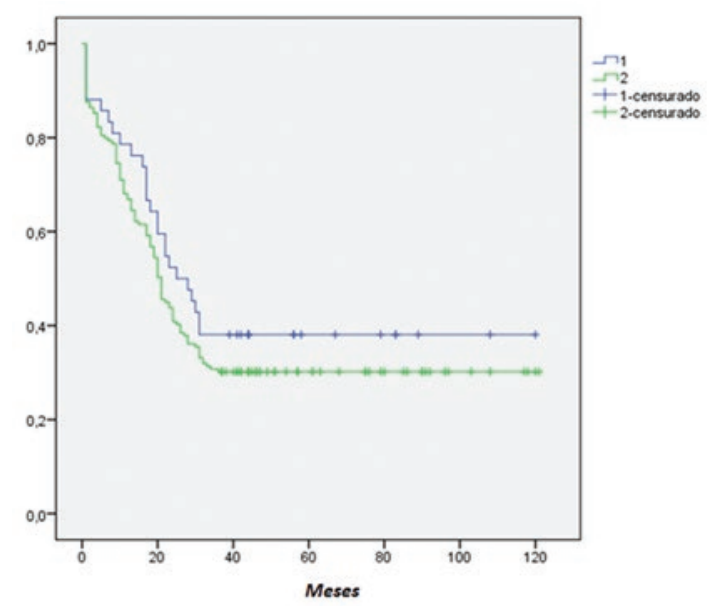

Figura 1. Supervivencia a 5 años $(p=0.254)$.

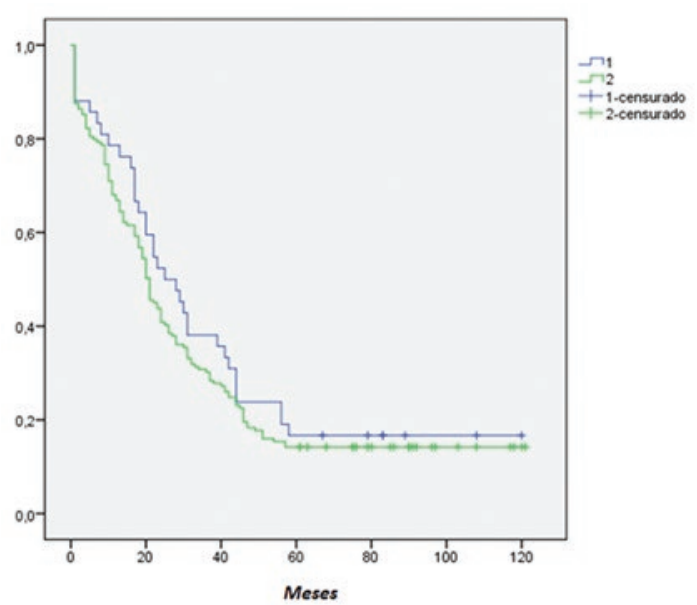

Figura 2. Supervivencia libre de enfermedad ( $p=0.348)$.

Mentha, et al. ${ }^{20}$ propusieron la resección diferida, pero de manera «inversa» 0 «hígado primero» (liver first). La evaluación de los resultados de dos de las tres estrategias (diferida clásica frente a simultánea) es el objetivo de este trabajo.

El $35.4 \%$ (212) de los pacientes sometidos a hepatectomía por metástasis hepáticas con intención curativa pertenecieron al grupo de las sincrónicas; porcentaje similar al reportado por otros autores, que oscila entre el 20 y el 40\% $\%^{2-5}$. De los 212 pacientes tratados, $149(70.3 \%)$ lo fueron de manera clásica y $63(29,7 \%)$ de manera simultánea. El tipo $C$ de Dukes fue el más frecuente en ambos grupos, y el número de resecciones colónicas fue mayor que el de las rectales, lo que es coincidente con otras experiencias. Algunos autores ${ }^{4,21-23}$ deciden la estrategia basándose en esto último; la localización rectal implica un mayor trauma quirúrgico y favorecería la elección de un procedimiento diferido. El número de hepatectomías 
mayores fue más alto en el grupo de tratamiento clásico $(p=0.005)$, lo que coincide con lo publicado por otros autores y confirmaría que en aquellos pacientes con mayor extensión tumoral se trataría de evitar las resecciones mayores ${ }^{4,23-27}$. Yoshioka, et al. ${ }^{3}$, por otro lado, reportan un porcentaje de resecciones simultáneas $(84.7 \%)$ superior al nuestro, con resultados alentadores, y recomiendan el abordaje simultáneo. El resto de los parámetros evaluados, tales como pérdida hemática, presencia de enfermedad extrahepática, margen de resección, etc., no mostraron diferencias significativas entre ambos grupos y son comparables a los de otras series. La estancia hospitalaria fue similar en ambos grupos ( $p=0.187$ ): 11.6 días para las resecciones simultáneas y 9.4 días para las diferidas, lo que confirma una de las principales ventajas de las resecciones simultáneas, ya que para el caso de las diferidas se requiere otro ingreso, otro procedimiento anestésico y, por supuesto, otro abordaje de la cavidad abdominal. Reddy, et al. ${ }^{4}$, comparando ambas estrategias, encuentran diferencias significativas entre ambas ( 9 vs. 14 días en las hepatectomías mayores y 8.5 vs. 14 días en las menores), y la corta estancia a favor de la resección simultánea implica beneficios económicos. La morbilidad y la mortalidad en ambos grupos no presentaron diferencias significativas ( $p=0.256$ y $p=0.508$, respectivamente). Cuando se analizó cada una de las complicaciones de las hepatectomías, notamos que la insuficiencia hepática fue mayor $(p=0.037)$ en el grupo de las diferidas; esta diferencia se justifica porque es también en las diferidas en las que hay un mayor número de hepatectomías mayores. Nosotros no incluimos en este trabajo las complicaciones inherentes a la primera cirugía en el abordaje clásico, pero podría inferirse que su incorporación hubiera mostrado un mayor número en el grupo de las diferidas. Reddy, et al. ${ }^{4}$ reportan que la morbilidad grave global en ambos grupos presentó diferencias significativas en aquellos pacientes que requirieron una hepatectomía mayor a favor de la estrategia diferida (36.1 vs. 17.6\%), mientras que no hubo diferencias cuando se compararon las menores; los mismos resultados expresan los estudios de Thelen, et al. ${ }^{28}$ y Bolton y Fuhrman ${ }^{29}$, por lo que estos autores recomiendan emplear las resecciones simultáneas solo en aquellos pacientes a los que se realizarán hepatectomías menores. En otro sentido, Martin, et al..$^{22}$, Capussotti, et al. ${ }^{30}$ y De Santibañes, et al. $^{31}$ muestran que cuando se comparan resecciones simultáneas y diferidas no hay diferencias en cuanto a complicaciones de origen hepático o colorrectal.

Los resultados en cuanto a supervivencia y supervivencia libre de enfermedad con ambas estrategias, como puede observarse en las figuras 1 y 2 , no mostraron diferencias, resultando coincidente con otras series $^{13}$. Lykoudis, et al. ${ }^{32}$, comparando las tres estrategias (colon first, simultánea y liver first) no encuentran diferencia a favor de ninguna, lo que reforzaría la indicación de las resecciones simultáneas.

Algunos factores se han relacionado con un mal pronóstico; la localización rectal del tumor primario ha sido asociada a peor pronóstico que la localización colónica, pero en nuestro análisis de esta variable no encontramos diferencias significativas ( $p=0.842)$. La presencia de ganglios positivos en la resección intestinal también se ha propuesto como un factor de mal pronóstico, pero el análisis de esta variable tampoco arrojó diferencia ( $p=0.147)$; cabría valorar si la relación entre ganglios positivos y totales, como se ha propuesto para diferentes tumores del aparato digestivo, podría tener implicancias pronósticas ${ }^{33}$.

Algunos autores refieren que la localización bilobar de las metástasis o un número mayor de tres lesiones tiene implicancias pronosticas negativas; los hallazgos en esta serie no coinciden con lo expresado por esos autores ( $p=0.203$ y $p=0.562$, respectivamente).

Este estudio presenta múltiples limitaciones. Una de ellas es que es un trabajo retrospectivo de datos recogidos prospectivamente; otra, tal vez la más importante, es la cantidad de años que abarca la serie, durante los cuales se aplicaron a los pacientes diferentes procedimientos de sección parenquimatosa y diversos esquemas de quimioterapia.

A manera de conclusión, nuestros resultados muestran que las resecciones simultáneas de metástasis hepáticas de origen colorrectal son un procedimiento con una morbimortalidad y una supervivencia similares a las de las resecciones realizadas de manera diferida, lo que representaría un beneficio para los pacientes desde el punto de vista de los efectos hepatotóxicos de los quimioterápicos modernos, así como una indudable mejora desde el punto de vista costo-beneficio. Sin embargo, quedaría una interrogante que requeriría ser respondida con más certidumbre: ¿tienen las hepatectomías mayores más morbimortalidad cuando se hacen de manera simultánea? La respuesta podría condicionar el ingreso de un grupo de pacientes a un protocolo de cirugía simultánea, aunque la tendencia parece ser avanzar hacia actitudes más agresivas ${ }^{34,35}$. 


\section{Responsabilidades éticas}

Protección de personas y animales. Los autores declaran que para esta investigación no se han realizado experimentos en seres humanos ni en animales.

Confidencialidad de los datos. Los autores declaran que han seguido los protocolos de su centro de trabajo sobre la publicación de datos de pacientes.

Derecho a la privacidad y consentimiento informado. Los autores declaran que en este artículo no aparecen datos de pacientes.

\section{Bibliografía}

1. Nari G, Moreno E, Feliu L, Azar R, Bonaparte F. Resecciones hepáticas sin tecnología de punta. Ocho años de experiencia. Rev Colomb Cir. 2011;26:48-55

2. Yin Z, Liu Ch, Chen Y, Bai Y, Shang Ch, Yin R, et al. Timing of hepatectomy in resectable synchronous colo-rectal liver metastases (SCRLM): simultaneous or delayes. Hepatology. 2013;57:2346-57.

3. Yoshioka R, Hasegawa K, Mise $Y$, Oba M, Aoki T, Sakamoto $Y$, et al Evaluation of safety and efficacy of simultaneous resection of primary colorectal cancer and synchronous liver metastases. Surgery. 2014 155:478-85.

4. Reddy S, Pawlik T, Zorzi D, Gleisner A, Ribero D, Assumpcao L, et al. Simultaneous resection of colorectal cancer and synchronous liver metastases: a multi-institutional analysis. Ann Surg Oncol. 2007;14:381-91.

5. Minagawa M, Yamamoto J, Miwa S, Sakamoto Y, Kokudo N, Kosuge T, et al. Selection criteria for simultaneous resection in patients with synchronous liver metastasis. Arch Surg. 2006;141:1006-12.

6. Bismuth $\mathrm{H}$. Surgical anatomy and anatomical surgery of the liver. World J Surg. 1982;6:3-9

7. González H, Figueras J. Practical questions in liver metastases of colorectal cancer: general principles of treatment. HPB. 2007;9:251-8.

8. Makuuchi M, Takayasu K, Takuma Y. Preoperative trans-catheter embolization of the portal venous branch for patients receiving extended lobectomy due to bile duct carcinoma. J Jpn Surg Assoc. 1984;45:1558-64.

9. Stolz A, Gagniere J, Dupre M, Rivoire M. Destruction locoregionale des metastases hepatiques d'origine colorectale. Place de la radiofrequence. Journal de Chirurgie Viscerale. 2014;151:534-46.

10. De Santibañes E, Clavien J. Playing play-doh to prevent postoperative liver failure. The "ALPPS" approach. Ann Surg. 2012;255:415-7.

11. Grundmann R. Current state of surgical treatment of liver metastases from colorectal cancer. Word J Gastrointest Surg. 2011;27:183-96.

12. Fahy B, Fischer $C$. Syncronous resection of colorectal primary and hepatic mestastasis. J Gastrointest Oncol. 2012;3:48-58.

13. Mayo S, Pulitano C, Marques H, Lamelas J, Wolfgang C, de Saussure W, et al. Surgical management of patients with synchronous colorectal liver matastases: a multicenter international analysis. J Am Coll Surg. 2013; 216:707-18.

14. Vauthey J, Pawlik T, Ribero D, Wu T, Zorzi D, Hoff P, et al. Chemotherapy regimen predicts steatohepatitis and an increase in 90 day mortality after surgery for hepatic colorectal metastases. J Clin Oncol. 2006;24:2065-72
15. Aloia T, Sebagh M, Plasse M, Karam V, Levi F, Giachetti S, et al. Liver histology and surgical outcomes after preoperative chemotherapy with fluorouracil plus oxiplatin in colorectal cancer liver metastases. J Clin Oncol. 2006;24:4983-90.

16. Karoui M, Penna C, Amin-Hashem M, Mitry E, Benoist S, Franc B, et al Influence of chemotherapy on the risk of major hepatectomy for colorectal liver metastases. Ann Surg. 2006;243:1-7.

17. Strasberg S, Phillips C. Use and dissemination of the Brisbane 2000 nomenclature of liver anatomy and resections. Ann Surg. 2013;257:377-82.

18. Dindo D, Demartines N, Clavien P. Classification of surgical complications. A new proposal with evaluation in a cohort of 6336 patients and results of survey. Ann Surg. 2004;240:205-13.

19. Balzan S, Belghiti J, Farges O, Ogata S, Sauvanet A, Delefosse D, et al. The 50-50 criteria on postoperative day 5: an accurate predictor of liver failure and death after hepatectomy. Ann Surg. 2005;242:824-8.

20. Mentha G, Roth AD, Terraz S, Giostra E, Gervaz P, Andrés A, et al. Liver first approach in the treatment of colorectal cancer with synchronous liver metastases. Dig Surg. 2008;25:430-5.

21. Martin RC $2^{\text {nd }}$, Augenstein V, Reuter N, Scoggins C, Mc Masters K. Simultaneous versus staged resection for synchronous colorectal cancer liver metastases. J Am Coll Surg. 2009:208:842-50.

22. Martin R, Paty P, Fong Y, Grace A, Cohen A, De Matteo R, et al. Simultaneous liver and colorectal resection are safe for synchronous colorectal liver metastases. J Am Coll Surg. 2003;197:23-41.

23. Tanaka K, Shimada H, Matsuo K, Nagano Y, Endo I, Sekido H, et al. Outcom after simultaneous colorectal and hepatic resection for colorectal cancer with synchronous metastases. Surgery. 2004:136:650-9.

24. Weber J, Bachellier P, Oussoultzoglou E, Jaek D. Simultaneous resection of colorectal primary tumour and syncronic liver metastases. $\mathrm{Br} \mathrm{J}$ Surg. 2003;90:956-62.

25. Turrini O, Viret F, Guiramand J, Lelong B, Bege T, Delpiero J. Strategies for the treatment of synchronous liver metastasis. Eur J Surg Oncol. 2007;33:735-40.

26. Luo Y, Wang L, Chen C, Chen D, Huang M, Huang Y, et al. Simultaneous liver and colorectal resections are safe for synchronous colorectal liver metastases. J Gastrointest Surg. 2010;14:1974-80.

27. Abbot D, Cantor S, Hu C, Aloia T, You Y, Nguyen S, et al. Optimizing clinical and economic outcomes of surgical therapy for patients with colorectal cancer and synchronous liver metastases. J Am Coll Surg. 2012;215:262-70.

28. Thelen A, Jonas S, Benckert C, Spinelli A, Lopez-Hänninen E, Rudolph B, et al. Simultaneous versus staged liver resection of synchronous liver metastases from colorectal cancer. Int J Colorectal Dis. 2007; 22(10):1269-76.

29. Bolton J, Fuhrman G. Survival after resection of multiple bilobar metastases from colorectal carcinoma. Ann Surg. 2000;231:743-51.

30. Capussotti L, Ferrero A, Vigano L. Timing of resection of liver metastases synchronous with colorectal surgery. Ann Surg Oncol. 2007:14:195-201.

31. De Santibañes E, Lasalle F, Mc Cormack L. Simultaneous colorectal and hepatic resections for colorectal cancer: postoperative and long-term outcomes. J Am Coll Surg. 2002;195:196-202.

32. Lykoudis P, O'Reilly D, Nastos K, Fussi G. Systematic review of surgical management of synchronous colorectal liver metastases. BJS. 2014; 101:605-12.

33. Nari G, Palacios O, López Ben S, Albiol M, Falgueras L, Castro Gutiérrez E, et al. Hilar cholangiocarcinoma: the number of positive nodes and positive node/total node ratio is a significant prognostic factor for survival. Cir Esp. 2014:92:247-53.

34. Karoui M, Vigano L, Goyer P, Ferrero A, Luciani A, Aglietta M, et al. Combined first stage hepatectomy and colorectal resection in a two stage hepatectomy strategy for bilobar synchronous liver metastases. $\mathrm{Br}$ J Surg. 2010;97:1354-62

35. Andres A, Mentha G, Adam R, Gerstel E, Skipenko O, Barroso E, et al. Surgical management of patients with colorectal cancer and simultaneous liver and lung metastases. BJS. 2015;102:691-9. 